\title{
Manipulation of Alcohol and Short-Chain Fatty Acids in the Metabolome of Commensal and Virulent Klebsiella pneumoniae by Linolenic Acid
}

\author{
Ryan Yuki Huang ${ }^{1,2}$, Deron Raymond Herr ${ }^{3}$ and Shabbir Moochhala ${ }^{3, *}$ \\ Canyon Crest Academy, San Diego, CA 92130, USA; rhuang53@gmail.com \\ 2 Department of Mechanical and Aerospace Engineering, University of California, San Diego, CA 92093, USA \\ 3 Department of Pharmacology, National University of Singapore, Singapore 117600, Singapore; \\ phcdrh@nus.edu.sg \\ * Correspondence: phcsmm@nus.edu.sg; Tel.: +65-8511-0112
}

Received: 9 April 2020; Accepted: 18 May 2020; Published: 21 May 2020

\begin{abstract}
Endogenous alcohol produced by the gut microbiome is transported via the bloodstream to the liver for detoxification. Gut dysbiosis can result in chronic excess alcohol production that contributes to the development of hepatic steatosis. The aim of this study was to examine whether linolenic acid can manipulate the production of harmful alcohol and beneficial short-chain fatty acids (SCFAs) in the metabolome of commensal Klebsiella pneumoniae (K. pneumoniae) and the virulent $K$. pneumoniae K1 serotype. Glucose fermentation by the K. pneumoniae K1 serotype yielded increased production of alcohol and decreased SCFAs (especially acetate and propionate) compared to those of commensal K. pneumoniae. However, the use of linolenic acid instead of glucose significantly reduced alcohol and increased SCFAs in the fermentation media of the K. pneumoniae K1 serotype. The work highlights the value of shaping the microbial metabolome using linolenic acid, which can potentially regulate the gut-liver axis for the prevention and treatment of alcohol-induced liver diseases.
\end{abstract}

Keywords: Klebsiella pneumoniae; linolenic acid; metabolome; microbiome; prebiotics

\section{Introduction}

The human gut microbiome consists of the entirety of the microbes that reside in the human gut [1]. These microbes aid in human innate immunity, metabolic functions, and overall wellbeing. However, dysbiosis in the gut microbiome can alter host-microbe interactions and yield detrimental effects for the host. The gut microbiome links and modulates the activity of other human organs through its axes, such as the gut-brain axis, the gut-lung axis, and the gut-liver axis. Thus, manipulation of the biological functions of microbes to alter the production of microbial metabolites in the gut can extend beyond local effects on the gut and may influence distant organ systems.

Certain bacteria residing in the human gut are associated with liver damage via the gut-liver axis. For instance, the high alcohol-producing W14 and TH1 strains of Gram-negative Klebsiella pneumoniae (K. pneumoniae) are proven to be major factors in contributing to non-alcoholic fatty liver diseases (NAFLD) [2]. Cytolysin-producing Enterococcus faecalis (E. faecalis), a Gram-positive bacterium, has been found in larger quantities in alcoholic hepatitis patients than their healthy counterparts [3]. Most notably, hypervirulent strains of the capsular serotypes $\mathrm{K} 1$ or $\mathrm{K} 2$ of K. pneumoniae are known to induce liver abscesses or bacteremia in patients. The literature has demonstrated that the K. pneumoniae $\mathrm{K} 1$ serotype in particular can induce primary liver abscesses and is a risk factor for metastasis [4]. The serotype has been shown to exhibit large amounts of a hypermucoviscous phenotype that enhances its ability to cause severe infections. There are two genes that are associated with its virulence. 
The first is the mucoviscosity-associated gene A (magA). The magA gene enables the ability to form a mucoviscous string and to obtain higher resistance to phagocytosis, which has been widely reported in Asia. The second virulence gene is the regulator of the mucoid phenotype A $(r m p A)$, which is associated with synthesizing polysaccharide capsules [4]. Additionally, it was demonstrated that the rmpA gene was significantly correlated with the hypermucoviscous phenotype and the development of liver abscesses using multivariate analysis [4]. While the etiology is not certain, the reports above clearly illustrate that the gut-liver axis plays a significant role in the development of liver diseases as virulent bacteria in the gut microbiome are correlated with liver damage.

The liver is a vital organ that helps with bile production and metabolization and bilirubin absorption and, most importantly, serves to provide first-pass metabolism to detoxify blood from the digestive system. As excess alcohol, which is consumed by humans or produced by K. pneumoniae and other gut bacteria, enters the liver through the bloodstream, it can lead to the formation of reactive oxygen species (ROS), or free radicals. These free radicals provoke the formation of toxic aldehydes, leading to severe liver abscesses. Current treatment methods for liver damage typically involve lifestyle changes, including weight management, abstinence from alcohol, and nutritional therapy. There are also drug therapies, including the administration of corticosteroids to decrease the cytokine response and pentoxifylline to increase $3^{\prime}, 5^{\prime}$-cyclic monophosphate (cAMP) concentrations [5]. However, administration of these drugs may yield more serious side effects, including liver injury and infection [6]. Furthermore, lifestyle changes and current drug therapies do not directly affect the progression of liver damage itself but, rather, they help manage and prevent possible adverse effects, including diabetes and cardiovascular issues. While antibiotic therapy represents a possible direct treatment option to eradicate high alcohol-producing K. pneumoniae, many antibiotic resistant $K$. pneumoniae strains have been identified [7]. Moreover, antibiotics lack the specificity to selectively kill harmful K. pneumoniae strains and would also eradicate commensal or beneficial bacteria in the human microbiome. Another possible method is the introduction of genetically modified K. pneumoniae with reduced virulence [8]; however, this treatment choice risks transferring K. pneumoniae genes to other microbes and/or host cells.

Even without genetic modifications, gut-residing microbes can naturally benefit the host. For instance, beneficial microbes may produce antimicrobial agents to combat pathogens, and probiotic bacteria may produce beneficial metabolites to help fight diseases and to alleviate dysbiosis in the microbiome. Probiotics have been defined by the Food and Agriculture Organization of the World Health Organization as live microorganisms that, when administered in sufficient amounts, provide a health benefit for the host [9]. It has been documented that short-chain fatty acids (SCFAs) are produced by these probiotic bacteria. Many SCFAs, such as acetic acid, butyric acid, or propionic acid, have anti-microbial and anti-inflammatory properties. SCFAs are also capable of decreasing inflammation via the inhibition of histone deacetylase (HDAC) [10]. Unlike other treatment options, probiotics do not require host immune activation and may not affect other commensal bacteria. While promising, the Food and Drug Administration (FDA) has not yet approved administering probiotic bacteria for the treatment of liver diseases. Probiotic bacteria in the human gut microbiome can metabolize glucose, fiber, or other prebiotics to produce a beneficial metabolome, a collection of fermentation metabolites including SCFAs [11]. Clinical studies of supplementing gut probiotic bacteria with prebiotics to induce SCFA production for the treatment of human diseases such as type 2 diabetes mellitus have been extensively conducted [12]. The FDA has established definitions for prebiotics and permits food manufacturers to self-affirm GRAS (generally recognized as safe) status for products ultimately labeled as prebiotics [9]. Prebiotics were originally defined by Gibson and Roberfroid in 1995 [13] as "nondigestible food ingredients that beneficially affect the host by selectively stimulating the growth and/or activity of one or a limited number of bacterial species already resident in the colon, and thus attempt to improve host health". This definition was later revised by Gibson et al [14,15] in 2004 as "a selectively fermented ingredient that allows specific changes, both in the composition and/or activity in the gastrointestinal microflora, that confer benefits upon host well-being and health". Glucose-based 
dietary fibers and non-carbohydrate substances including polyunsaturated fatty acid (PUFA) have been used as prebiotics for gut bacteria [16]. The use of prebiotics to naturally manipulate the metabolism of commensal or virulent $K$. pneumoniae may be a non-genetic approach to shift the dysbiotic metabolome to a healthier metabolome in the gut. In this study, we treated commensal K. pneumoniae and the K. pneumoniae K1 serotype with glucose or linolenic acid. The amounts of alcohol and major SCFAs in the media of K. pneumoniae incubated with glucose and linolenic acid were compared.

\section{Materials and Methods}

\subsection{Bacterial Culture and Fermentation}

Chemicals were purchased from Thermo Fisher Scientific (Fair Lawn, NJ, USA) or companies as indicated. A K. pneumoniae K1 (1084) strain [4] isolated from patients with liver abscesses was obtained from Dr. Ying-Tsong Chen, Institute of Genomics and Bioinformatics, National Chung Hsing University, Taiwan. A commensal K. pneumoniae C strain was isolated from the fecal samples of healthy subjects and identified by $16 \mathrm{~S}$ ribosomal RNA(rRNA) gene sequencing (Supplementary Figure S1). K. pneumoniae bacteria were cultured in tryptic soy broth (TSB) media to an absorbance at $600 \mathrm{~nm}$ [optical density $(\mathrm{OD})_{600}$ ] of 1.0. The bacterial samples were harvested by centrifugation at $5000 \times g$ for $10 \mathrm{~min}$. Then, they were washed with and suspended in phosphate buffered saline (PBS). For fermentation, the bacteria $\left(10^{5}\right.$ colony-forming unit $\left.(\mathrm{CFU}) / \mathrm{mL}\right)$ were incubated in rich media ( $10 \mathrm{~g} / \mathrm{L}$ yeast extract (Biokar Diagnostics, Beauvais, France), $3 \mathrm{~g} / \mathrm{L} \mathrm{TSB}, 2.5 \mathrm{~g} / \mathrm{L} \mathrm{K}{ }_{2} \mathrm{HPO}_{4}$, and $1.5 \mathrm{~g} / \mathrm{L}$ $\mathrm{KH}_{2} \mathrm{PO}_{4}$ ), in the absence or presence of $20 \mathrm{~g} / \mathrm{L}$ glucose or linolenic acid under anaerobic conditions using the Gas-Pak at $37^{\circ} \mathrm{C}$. Rich media with $20 \mathrm{~g} / \mathrm{L}$ glucose (G7021) or linolenic acid (L2376) (Sigma, St. Louis, MO, USA) without bacteria were used as controls. The samples were cultured in a Biosafety Level 2 (BSL-2) facility in America Diagnosis Inc., San Diego.

\subsection{Alcohol Detected by Gas Chromatographic Mass Spectrometry (GC-MS)}

The analysis of the alcohol in each $100 \mu \mathrm{L}$ sample was conducted in an Agilent 5973 mass selective detector, interfaced to an Agilent 6890 gas chromatography with an Agilent 7694 headspace sampler and a HP 5MS column $(25 \mathrm{~m} \times 0.25 \mathrm{~mm} \times 0.25 \mu \mathrm{m})$, which were all found at HT Labs Inc., San Diego. The flow rate of the carrier gas helium was set at $1 \mathrm{~mL} / \mathrm{min}$. A peak in the GC chromatograph with a retention time of $2.39 \mathrm{~min}$ was identified as alcohol and this was further confirmed by MS. Peak areas were used for quantitation. A five-point calibration curve covering the alcohol concentration range of $0-2 \mathrm{~g} / \mathrm{L}$ was constructed. Alcohol-free samples contained a spike with alcohol at a concentration of $0.05,0.1,0.5,1$, and $2 \mathrm{~g} / \mathrm{L}$ and were analyzed in triplicate.

\subsection{SCFA Identification by GC-MS}

K. pneumoniae $\mathrm{K} 1$ or commensal K. pneumoniae $\mathrm{C}\left(10^{5} \mathrm{CFU} / \mathrm{mL}\right)$ bacteria were incubated in rich media with $2 \%$ glucose or linolenic acid for 3 days. The fermentation media was centrifuged at $5000 \times \mathrm{g}$ to remove bacteria and filtered through $0.2 \mu \mathrm{m}$ filters. The fermentation media $(100 \mu \mathrm{l})$ were separately subjected to liquid-liquid extraction with ethyl acetate $(1: 1, v / v)$ for $10 \mathrm{~min}$ (Residue Analysis OmniSolv, Millipore, Billerica, MA) after adding an internal standard $\left(0.1 \mathrm{mg} / \mathrm{mL}{ }^{2} \mathrm{H} 7\right.$-butyric acid, C/D/N Isotopes, Quebec, Canada), followed by acidification with $0.5 \%$ ortho-phosphoric acid and saturation with sodium chloride $[17,18]$. GC-MS analysis for SCFAs in the aqueous phase was performed using an Agilent 5890 Series II GC, coupled with a 5971 MS detector (Agilent Technologies, Inc., Palo Alto, CA) at the Sanford Burnham Prebys Cancer Metabolism Core, San Diego. A 70-eV electron beam was used for ionization. SCFAs in the fermentation media were quantified by a calibration curve made from six non-zero levels using the 500-, 1,000-, 2,000-, 5,000-, and 10,000-fold dilutions of the Free Fatty Acids Test Standard (Restek Corporation, Bellefonte, PA) [19] which contained six SCFAs (acetate, butyrate, propionate, valerate, isobutyrate, and isovalerate). Those SCFAs with higher levels than the background signals were calculated. 


\subsection{Statistical Analysis}

Data analysis was performed using a Student $t$-test. The $p$-values of $<0.05\left(^{*}\right),<0.01\left({ }^{* *}\right)$, and $\left.<0.001{ }^{* * *}\right)$ were considered significant. The mean \pm standard deviation (SD) was calculated for at least three independent experiments.

\section{Results}

\subsection{Alcohol Production in K. pneumoniae}

Dietary fibers can be fermentatively converted to glucose by gut bacteria. Both dietary fibers [20] and glucose [21] have been used as substances to promote the SCFA products by the fermentation of gut bacteria. Thus, the ability of glucose $\left(\mathrm{C}_{6} \mathrm{H}_{12} \mathrm{O}_{6}\right)$ and linolenic acid $\left(\mathrm{C}_{18} \mathrm{H}_{30} \mathrm{O}_{2}\right)$ to induce the fermentation of commensal K. pneumoniae and the K. pneumoniae $\mathrm{K} 1$ serotype were examined. The fermentation of K. pneumoniae produced detectable alcohol, and its MS spectrum was shown in Figure 1 and concentration was quantified using GC-MS. As shown in Figure 1, in the presence of $20 \mathrm{~g} / \mathrm{L}$ glucose, the K. pneumoniae $\mathrm{K} 1$ serotype $(149 \pm 23 \mu \mathrm{mol} / \mathrm{mL})$ produced a higher amount of alcohol than the commensal K. pneumoniae strain $(94 \pm 12 \mu \mathrm{mol} / \mathrm{mL})$. When the carbon source was changed from glucose to linolenic acid, the alcohol production of the K. pneumoniae K1 serotype was considerably reduced. This result indicates that linolenic acid can be used as a prebiotic means of reducing the alcohol production of the K. pneumoniae K1 serotype.

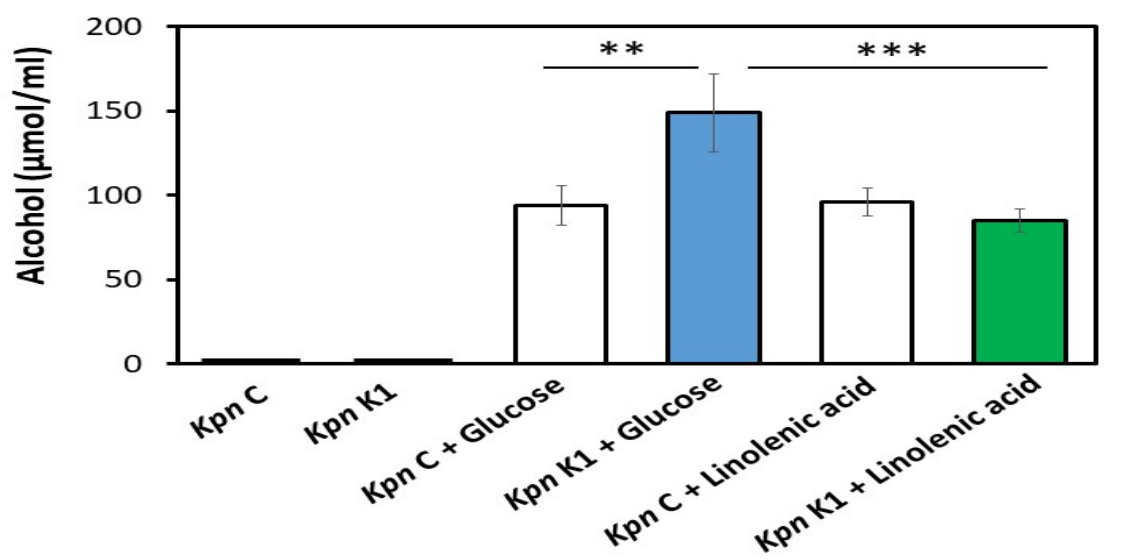

Figure 1. Alcohol production from glucose or linolenic acid fermentation of commensal K. pneumoniae (Kpn) C and virulent Kpn K1 strain. Kpn C or Kpn $\mathrm{K} 1$ bacteria $\left(10^{5} \mathrm{CFU} / \mathrm{mL}\right)$ were incubated in rich media in the absence or presence of $20 \mathrm{~g} / \mathrm{L}$ glucose or linolenic acid for 3 days. The alcohol in the fermentation media was detected by HP6890 GC-MS with a retention time of $2.39 \mathrm{~min}$. Alcohol concentrations ( $\mu \mathrm{mol} / \mathrm{mL}$ ) in rich media of $K p n \mathrm{C}$ alone, $K p n \mathrm{~K} 1$ alone, $K p n C$ with glucose, Kpn K1 with glucose, Kpn C with linolenic acid, and Kpn K1 with linolenic acid were determined. The mean \pm SD from three independent experiments and $p$-values of $<0.01\left({ }^{* *}\right)$ and $<0.001\left({ }^{* * *}\right)$ via a Student $t$-test were calculated and denoted accordingly.

\subsection{SCFA Production Enhanced by Linolenic Acid}

Six SCFAs were chosen to establish the calibration curves for the quantification of the SCFAs in the fermentation media of K. pneumoniae by GC-MS analysis. As shown in Figure 2, the incubation of commensal K. pneumoniae with $20 \mathrm{~g} / \mathrm{L}$ glucose generated four detectable SCFAs (acetate, propionate, isobutyrate, and butyrate) in the fermentation media. Valerate and isovalerate were not calculated as their levels were lower than the background signals in the GC-MS spectra. The acetate, propionate, isobutyrate, and butyrate were detected in a total ion chromatogram with retention times of 6.0, 8.2, 9.1, and $10.2 \mathrm{~min}$, respectively (Figure 2A). The mass spectra of four detectable SCFAs with major fragments are shown in Figure 2B. The results indicate that commensal K. pneumoniae is a fermenting 
gut bacterium. Although SCFAs were detected following the glucose fermentation of the K. pneumoniae $\mathrm{K} 1$ serotype, their amounts were markedly lower than those in the glucose fermentation of commensal K. pneumoniae (Table 1). Most importantly, the amounts of four major SCFAs (acetate, propionate, isobutyrate, and butyrate) produced by the K. pneumoniae K1 serotype were significantly increased when $20 \mathrm{~g} / \mathrm{L}$ glucose was replaced by $20 \mathrm{~g} / \mathrm{l}$ linolenic acid (Table 1). For example, $1.0 \pm 0.1$ or $66 \pm 5.0 \mu \mathrm{M}$ propionate was produced in the media of glucose or linolenic acid fermentation by the K. pneumoniae K1 serotype, respectively. Taken together, the data in Figures 1 and 2 demonstrate that linolenic acid can lower alcohol production and increase SCFA production during the fermentation of the K. pneumoniae $\mathrm{K} 1$ serotype. These data also highlight the ability of linolenic acid to modulate the metabolome of K. pneumoniae fermentation.

B

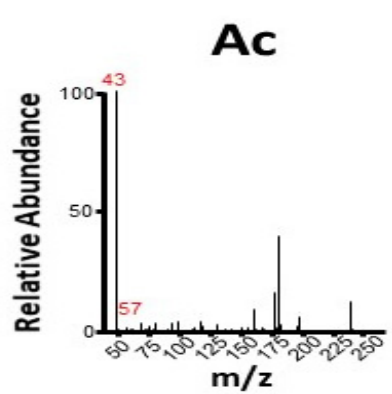

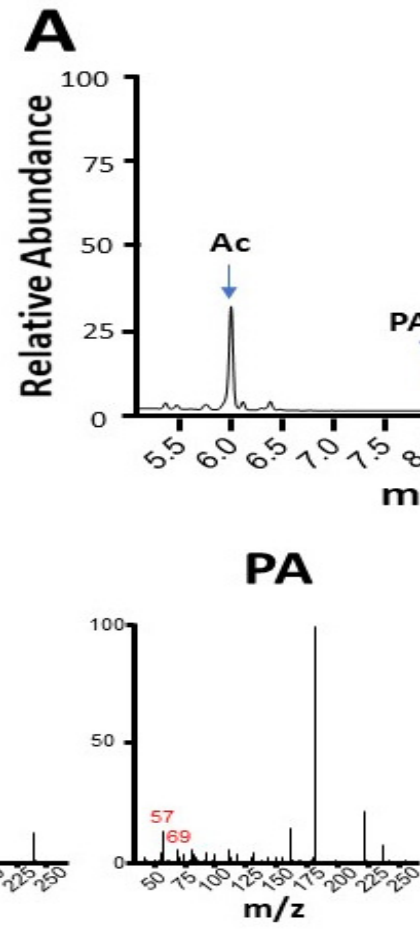

BA

Iso-BA
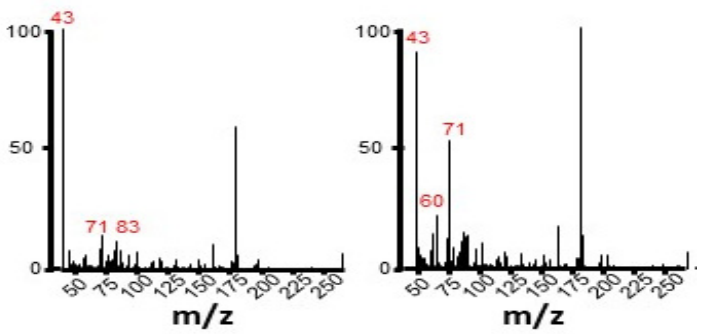

Figure 2. Production of short-chain fatty acids (SCFAs) by glucose fermentation of K. pneumoniae C strain. (A) Total ion chromatogram for separation of the mixture of SCFAs containing acetate (Ac), propionate (PA), isobutyrate (iso-BA), and butyrate (BA) was displayed by running the GC-MS analysis. (B) The mass spectra for acetate, propionate, isobutyrate, and butyrate with their corresponding molecular ions $(\mathrm{m} / \mathrm{z})$ are shown.

Table 1. Enhancement of SCFA production of K. pneumoniae (Kpn) K1 by linolenic acid fermentation.

\begin{tabular}{ccccc}
\hline SCFAs & $\begin{array}{c}\text { Kpn C } \\
\text { /Glucose }\end{array}$ & $\begin{array}{c}\text { Kpn C } \\
\text { /Linolenic acid }\end{array}$ & $\begin{array}{c}\text { Kpn } \mathbf{K 1} \\
\text { /Glucose }\end{array}$ & $\begin{array}{c}\text { Kpn } \mathbf{K 1} \\
\text { / Linolenic acid }\end{array}$ \\
\hline Acetate $(\mathrm{mM})$ & $1.3 \pm 0.1$ & $2.8^{*} \pm 0.3$ & $0.6 \pm 0.02$ & $9.0^{* *} \pm 1.1$ \\
Propionate $(\mu \mathrm{M})$ & $6.0 \pm 0.7$ & $15.0^{*} \pm 2.0$ & $1.0 \pm 0.1$ & $66.0^{* * *} \pm 5.0$ \\
Isobutyrate $(\mu \mathrm{M})$ & $20.9 \pm 3.2$ & $22.5 \pm 4.5$ & $18.5 \pm 3.3$ & $24.5^{*} \pm 4.2$ \\
\hline Butyrate $(\mu \mathrm{M})$ & $25.1 \pm 4.5$ & $27.2 \pm 4.2$ & $21.5 \pm 3.8$ & $30.1^{* *} \pm 4.2$ \\
\hline
\end{tabular}

The levels of acetate, propionate, isobutyrate, and butyrate produced by glucose and linolenic acid fermentation of $\mathrm{Kpn} \mathrm{C}$ and $\mathrm{Kpn} \mathrm{K} 1$ strains are shown. Data depict the mean $\pm \mathrm{SD}$ of three separate experiments. The $p$-values of $\left.<0.05\left(^{*}\right),<0.01{ }^{(* *}\right)$, and $<0.001\left(^{* * *}\right)$ via a Student $t$-test $($ Kpn C/glucose vs. Kpn C/linolenic acid; Kpn K1/glucose vs. Kpn K1/linolenic acid) are denoted. 


\section{Discussion}

A well-balanced diet rich in fibers and unsaturated fats leads to an increase in anti-inflammatory bacterial taxa such as Bifidobacteria [22]. The data in this study demonstrate for the first time that linolenic acid, a polyunsaturated $\omega-3$ fatty acid, can lower alcohol production yet induce an increase in SCFAs in the fermentation process of the virulent K. pneumoniae K1 serotype. Linolenic acid and its derivatives, or linolenic acid-rich foods such as flaxseed, pumpkin, and walnut oils, may function as natural prebiotics to lower the virulence of K. pneumoniae. The findings here also suggest that the treatment of liver abscesses with SCFAs may be able to minimize the cytotoxicity induced by high levels of alcohol in the gut. However, SCFAs with relatively short metabolic half-lives [23] may limit their therapeutic effects for the treatment of alcohol-induced liver diseases. Acetate in a $\mathrm{mM}$ range was detected in vitro in the fermentation media of K. pneumoniae. However, it was known that probiotic intakes may be able to only generate SCFAs in the blood at concentrations in the $\mu \mathrm{M}$ range [24]. Synthesis of an SCFA derivative with a longer half-life may increase the stability of SCFAs in vivo. For example, several propionic acid derivatives have been developed as pharmaceutic aids. These derivatives include drugs with brand names, such as Dermaclens, Transitonyl, Wound Klense, and Andriodermol [25]. SCFAs have been shown to exert crucial physiological effects on several human organs and have anti-inflammatory properties in numerous contexts. For example, butyrate produced by gut microbiota via the gut-brain axis is effective in reducing inflammation and pain in irritable bowel syndrome patients [26]. SCFAs, especially acetate, can significantly reverse ROS production in mesangial cells, and this can be recapitulated by treatment with an agonist for the cognate G protein-coupled receptor (GPCR), free fatty acid receptor 2 (FFAR2) [27]. Furthermore, dietary SCFA (acetate, propionate, and butyrate) intake improves liver metabolism by acting on FFAR3 [28]. Although the literature has indicated that increased levels of propionate promote liver gluconeogenesis in mice [29], results from a clinical study illustrated that propionate supplementation remarkably reduced intrahepatocellular lipid content [30].

K. pneumoniae is a commensal bacterium in the human gut microbiome, and gut-resident $\mathrm{K}$. pneumoniae strains have been shown to possess genotypes similar to those strains detected in liver abscesses and bacteremia [31]. Future works will include sequencing of the genome of K. pneumoniae $\mathrm{C}$ and comparing the expression of possible virulence genes with the K. pneumoniae $\mathrm{K} 1$ serotype. Multiple virulence genes in the K. pneumoniae $\mathrm{K} 1$ serotype significantly contribute to the development of liver abscesses [4]. Although it has been reported that certain gut bacteria can increase blood alcohol levels and induce liver disease [2,3], it is still unclear if commensal or virulent K. pneumoniae can yield alcohol via fermentation. Linolenic acid is a PUFA. Results from previous studies have demonstrated that linolenic acid can influence the growth and adhesion of probiotic lactic acid bacterial strains [32]. In addition, molecules with multiple carbon atoms, such as polyethylene glycerol (PEG), have been used as prebiotics to provide carbon sources for bacterial fermentation [18]. Not only intestinal commensal bacteria but also mammalian cells metabolize linolenic acid; these mammalian cells have a unique degradation pathway that yields unique metabolites. After absorption, linolenic acid can be metabolized into eicosapentaenoic acid (EPA), n-3 docosapentaenoic acid (n-3 DPA), and docosahexaenoic acid (DHA) through elongation of the carbon chain in the liver [33]. Thus, it is worth investigating how much SCFAs, EPA, n-3 DPA, and DHA can be produced in the gut when linolenic acid is used as an oral supplement. Although the original definition of a prebiotic was revised in 2004 [14], a panel of experts in microbiology, nutrition, and clinical research was convened by the International Scientific Association for Probiotics and Prebiotics in 2016 to review the definition and scope of prebiotics. The panel updated the definition of a prebiotic as a substrate that is selectively utilized by host microorganisms conferring a health benefit. This definition expands prebiotics to include non-carbohydrate substances including PUFA and conjugated linoleic acid (CLA), applications to body sites other than the gastrointestinal tract, and diverse categories other than food [16]. DHA derived from linolenic acid can exert a positive action by reverting the microbiome composition and increasing the production of anti-inflammatory compounds, like SCFAs, in inflammatory bowel 
disease [34]. Although our results demonstrated that linolenic acid can be fermented by gut bacteria to trigger the production of beneficial SCFAs in vitro, additional scientific and clinical studies may be required to validate the prebiotic activity of linolenic acid based on the three criteria described by Gibson et al, 2004 [14,15].

Blood alcohol concentration above $11 \mu \mathrm{mol} / \mathrm{mL}$ has been used to predict serum alanine aminotransferase (ALT) and aspartate aminotransferase (AST), two enzymes commonly used as biomarkers for liver damage [35]. Two high alcohol-producing W14 and TH1 strains of K. pneumoniae can produce more than $30 \mu \mathrm{mol} / \mathrm{mL}$ alcohol in a $12 \mathrm{~h}$ in vitro culture [2]. The results of this study showed that glucose fermentation of the K. pneumoniae $\mathrm{K} 1$ serotype for 3 days yielded approximately 149 $\mu \mathrm{mol} / \mathrm{mL}$ alcohol in the media, suggesting that the K. pneumoniae $\mathrm{K} 1$ serotype is a high alcohol-producing strain. It has been documented that alcohol consumption can cause a lower abundance of Bacteroidetes and a higher abundance of Proteobacteria in the human gut microbiome [36]. Thus, the interaction of alcohol-producing K. pneumoniae with Bacteroidetes and Proteobacteria can be explored. Enzymes in the pathway of the glucose fermentation of gut bacteria have been well characterized [37]. However, the enzymes involved in the conversion of linolenic acid to alcohol during the fermentation of $K$. pneumoniae have not been identified. Furthermore, the in vivo efficacy of using linolenic acid as a supplement to ameliorate alcohol-induced liver damage has not yet been determined.

The primary complication of using antibiotic therapy to eliminate virulent K. pneumoniae is the possibility of developing resistant bacterial strains. The data in this study emphasize the significance of using linolenic acid as a potential prebiotic for the production of beneficial metabolites during bacterial fermentation. Vaccines for new microbial strains cannot be administered on time during outbreaks. Humans host tremendous amounts of commensal bacteria in the microbiome at all times. Boosting the fermentation activity of these commensal bacteria by using "prebiotics" to augment a beneficial metabolome directly provides a readily available solution for the treatment of infections. It is estimated that there are five million annual cases of alcoholic liver diseases in the United States [38], with a mortality rate ranging from $30 \%$ to $50 \%$ [39]. Using prebiotics as supplements may impact these patients by shaping the metabolome of the virulent $K$. pneumoniae by promoting the production of beneficial SCFAs instead of alcohol.

Supplementary Materials: The following are available online at http://www.mdpi.com/2076-2607/8/5/773/s1, Supplementary Figure S1. 16S ribosomal RNA (rRNA) sequence of human isolates of K. pneumoniae using the 16S rRNA 41 forward (F) 5'-GAG TTT GAT CCT GGC TCA-3' and reverse (R) 5'-ACG GCT AAC TTG TTA CGA CT-3' 42 primers.

Author Contributions: R.Y.H. designed and performed experiments, and wrote this manuscript. D.R.H. assisted experimental designs and edited the manuscript. S.M. provided knowledge of gut microbiome and edited the manuscript. All authors have read and agreed to the published version of the manuscript.

Funding: This research received no external funding.

Acknowledgments: We gratefully appreciate Professor Renkun Chen at Department of Mechanical and Aerospace Engineering for providing an Internship for R.Y.H. at University of California, San Diego (UCSD) to conduct research. We appreciate Dr. Yang Jiang for providing chemicals and materials for experiments and helping in culturing K. pneumoniae K1 serotype under a BSL-2 faculty in America Diagnosis, Inc., San Diego. We thank Dr. David A Scott at the Sanford Burnham Prebys Cancer Metabolism Core, San Diego for GC-MS analysis. We also acknowledge valuable comments from judges for R.Y.H's presentation at the 2020 Greater San Diego Science and Engineering Fair.

Conflicts of Interest: All co-authors do not have any financial conflicts of interest and the funder has not played any decision-making role in the research.

\section{References}

1. Schwabe, R.F.; Greten, T.F. Gut Microbiome in HCC-Mechanisms, Diagnosis and Therapy. J. Hepatol. 2020, 72, 230-238. [CrossRef] [PubMed]

2. Yuan, J.; Chen, C.; Cui, J.; Lu, J.; Yan, C.; Wei, X.; Zhao, X.; Li, N.; Li, S.; Xue, G.; et al. Fatty Liver Disease Caused by High-Alcohol-Producing Klebsiella pneumoniae. Cell Metab. 2019, 30, 675-688. [CrossRef] [PubMed] 
3. Duan, Y.; Llorente, C.; Lang, S.; Brandl, K.; Chu, H.; Jiang, L.; White, R.C.; Clarke, T.H.; Nguyen, K.; Torralba, M.; et al. Bacteriophage targeting of gut bacterium attenuates alcoholic liver disease. Nature 2019, 575, 505-511. [CrossRef] [PubMed]

4. $\quad$ Lin, A.C.; Liao, T.L.; Lin, Y.C.; Lai, Y.C.; Lu, M.C.; Chen, Y.T. Complete genome sequence of Klebsiella pneumoniae 1084, a hypermucoviscosity-negative K1 clinical strain. J. Bacteriol. 2012, 194, 6316. [CrossRef]

5. Chen, S.S.; Block, B.S.; Chan, P.J. Pentoxifylline attenuates HPV-16 associated necrosis in placental trophoblasts. Arch. Gynecol. Obstet. 2015, 291, 647-652. [CrossRef]

6. Vargas, J.I.; Arrese, M.; Shah, V.H.; Arab, J.P. Use of Statins in Patients with Chronic Liver Disease and Cirrhosis: Current Views and Prospects. Curr. Gastroenterol. Rep. 2017, 19, 43. [CrossRef]

7. Navon-Venezia, S.; Kondratyeva, K.; Carattoli, A. Klebsiella pneumoniae: A major worldwide source and shuttle for antibiotic resistance. FEMS Microbiol. Rev. 2017, 41, 252-275. [CrossRef]

8. Jayol, A.; Poirel, L.; Villegas, M.V.; Nordmann, P. Modulation of mgrB gene expression as a source of colistin resistance in Klebsiella oxytoca. Int. J. Antimicrob. Agents 2015, 46, 8-10. [CrossRef]

9. Venugopalan, V.; Shriner, K.A.; Wong-Beringer, A. Regulatory oversight and safety of probiotic use. Emerg. Infect. Dis. 2010, 16, 1661-1665. [CrossRef]

10. Tong, X.; Yin, L.; Giardina, C. Butyrate suppresses cox-2 activation in colon cancer cells through HDAC inhibition. Biochem. Biophys. Res. Commun. 2004, 317, 463-471. [CrossRef]

11. Pilla, R.; Suchodolski, J.S. The Role of the Canine Gut Microbiome and Metabolome in Health and Gastrointestinal Disease. Front. Vet. Sci. 2020, 6, 498. [CrossRef] [PubMed]

12. Tonucci, L.B.; Olbrich Dos Santos, K.M.; Licursi de Oliveira, L.; Rocha Ribeiro, S.M.; Duarte Martino, H.S. Clinical application of probiotics in type 2 diabetes mellitus: A randomized, double-blind, placebo-controlled study. Clin. Nutr. 2017, 36, 85-92. [CrossRef] [PubMed]

13. Gibson, G.R.; Roberfroid, M.B. Dietary modulation of the human colonic microbiota: Introducing the concept of prebiotics. J. Nutr. 1995, 125, 1401-1412. [CrossRef] [PubMed]

14. Gibson, G.R.; Probert, H.M.; Loo, J.V.; Rastall, R.A.; Roberfroid, M.B. Dietary modulation of the human colonic microbiota: Updating the concept of prebiotics. Nutr. Res. Rev. 2004, 17, 259-275. [CrossRef] [PubMed]

15. Gibson, G.R.; Scott, K.P.; Rastall, R.A.; Tuohy, K.M.; Hotchkiss, A.; Dubert-Ferrandon, A.; Garau, M.; Murphy, E.F.; Saulnier, D.; Loh, G.; et al. Dietary prebiotics: Current status and new definition. Food Sci. Technol. Bull. Funct. Foods 2010, 7, 1-19. [CrossRef]

16. Gibson, G.R.; Hutkins, R.; Sanders, M.E.; Prescott, S.L.; Reimer, R.A.; Salminen, S.J.; Scott, K.; Stanton, C.; Swanson, K.S.; Cani, P.D.; et al. Expert consensus document: The International Scientific Association for Probiotics and Prebiotics (ISAPP) consensus statement on the definition and scope of prebiotics. Nat. Rev. Gastroenterol. Hepatol. 2017, 14, 491-502. [CrossRef]

17. Aghazadeh, M.; Engelberth, A.S. Techno-economic analysis for incorporating a liquid-liquid extraction system to remove acetic acid into a proposed commercial scale biorefinery. Biotechnol. Prog. 2016, 32, 971-977. [CrossRef]

18. Kao, M.S.; Huang, S.; Chang, W.L.; Hsieh, M.F.; Huang, C.J.; Gallo, R.L.; Huang, C.M. Microbiome precision editing: Using PEG as a selective fermentation initiator against methicillin-resistant Staphylococcus aureus. Biotechnol. J. 2017, 12. [CrossRef]

19. Sharma, V.; Smolin, J.; Nayak, J.; Ayala, J.E.; Scott, D.A.; Peterson, S.N.; Freeze, H.H. Mannose Alters Gut Microbiome, Prevents Diet-Induced Obesity, and Improves Host Metabolism. Cell Rep. 2018, 24, 3087-3098. [CrossRef]

20. Meyer, D. Health benefits of prebiotic fibers. Adv. Food Nutr. Res. 2015, 74, 47-91. [CrossRef]

21. Egert, M.; de Graaf, A.A.; Maathuis, A.; de Waard, P.; Plugge, C.M.; Smidt, H.; Deutz, N.E.; Dijkema, C.; de Vos, W.M.; Venema, K. Identification of glucose-fermenting bacteria present in an in vitro model of the human intestine by RNA-stable isotope probing. FEMS Microbiol. Ecol. 2007, 60, 126-135. [CrossRef] [PubMed]

22. Shokryazdan, P.; Faseleh Jahromi, M.; Navidshad, B.; Liang, J.B. Effects of prebiotics on immune system and cytokine expression. Med. Microbiol. Immunol. 2017, 206, 1-9. [CrossRef] [PubMed]

23. Maniar, K.; Singh, V.; Moideen, A.; Bhattacharyya, R.; Chakrabarti, A.; Banerjee, D. Inhalational supplementation of metformin butyrate: A strategy for prevention and cure of various pulmonary disorders. Biomed. Pharmacother. 2018, 107, 495-506. [CrossRef] [PubMed] 
24. Parker, A.; Fonseca, S.; Carding, S.R. Gut microbes and metabolites as modulators of blood-brain barrier integrity and brain health. Gut Microbes 2020, 11, 135-157. [CrossRef]

25. Zhang, X.Z.; Chen, W.B.; Wu, X.; Zhang, Y.W.; Jiang, Y.M.; Meng, Q.X.; Zhou, Z.M. Calcium propionate supplementation improves development of rumen epithelium in calves via stimulating $G$ protein-coupled receptors. Animal 2018, 12, 2284-2291. [CrossRef]

26. Moser, G.; Fournier, C.; Peter, J. Intestinal microbiome-gut-brain axis and irritable bowel syndrome. Wien. Med. Wochenschr. 2018, 168, 62-66. [CrossRef]

27. Huang, W.; Guo, H.L.; Deng, X.; Zhu, T.T.; Xiong, J.F.; Xu, Y.H.; Xu, Y. Short-Chain Fatty Acids Inhibit Oxidative Stress and Inflammation in Mesangial Cells Induced by High Glucose and Lipopolysaccharide. Exp. Clin. Endocrinol. Diabetes 2017, 125, 98-105. [CrossRef]

28. Shimizu, H.; Masujima, Y.; Ushiroda, C.; Mizushima, R.; Taira, S.; Ohue-Kitano, R.; Kimura, I. Dietary short-chain fatty acid intake improves the hepatic metabolic condition via FFAR3. Sci. Rep. 2019, 9, 16574. [CrossRef]

29. Wong, J.M.; de Souza, R.; Kendall, C.W.; Emam, A.; Jenkins, D.J. Colonic health: Fermentation and short chain fatty acids. J. Clin. Gastroenterol. 2006, 40, 235-243. [CrossRef]

30. Chambers, E.S.; Viardot, A.; Psichas, A.; Morrison, D.J.; Murphy, K.G.; Zac-Varghese, S.E.; MacDougall, K.; Preston, T.; Tedford, C.; Finlayson, G.S.; et al. Effects of targeted delivery of propionate to the human colon on appetite regulation, body weight maintenance and adiposity in overweight adults. Gut 2015, 64, 1744-1754. [CrossRef]

31. Paczosa, M.K.; Mecsas, J. Klebsiella pneumoniae: Going on the Offense with a Strong Defense. Microbiol. Mol. Biol. Rev. 2016, 80, 629-661. [CrossRef] [PubMed]

32. Kankaanpää, P.E.; Salminen, S.J.; Isolauri, E.; Lee, Y.K. The influence of polyunsaturated fatty acids on probiotic growth and adhesion. FEMS Microbiol. Lett. 2001, 194, 149-153. [CrossRef] [PubMed]

33. Nagatake, T.; Kunisawa, J. Emerging roles of metabolites of $\omega 3$ and $\omega 6$ essential fatty acids in the control of intestinal inflammation. Int. Immunol. 2019, 31, 569-577. [CrossRef] [PubMed]

34. Costantini, L.; Molinari, R.; Farinon, B.; Merendino, N. Impact of Omega-3 Fatty Acids on the Gut Microbiota. Int. J. Mol. Sci. 2017, 18, 2645. [CrossRef]

35. Webster, G.F.; Webster, T.G.; Grimes, L.R. Laboratory tests in patients treated with isotretinoin: Occurrence of liver and muscle abnormalities and failure of AST and ALT to predict liver abnormality. Dermatol. Online J. 2017, 23, 77.

36. Engen, P.A.; Green, S.J.; Voigt, R.M.; Forsyth, C.B.; Keshavarzian, A. The Gastrointestinal Microbiome: Alcohol Effects on the Composition of Intestinal Microbiota. Alcohol. Res. 2015, 37, 223-236.

37. Andreesen, J.R.; Schaupp, A.; Neurauter, C.; Brown, A.; Ljungdahl, L.G. Fermentation of glucose, fructose, and xylose by Clostridium thermoaceticum: Effect of metals on growth yield, enzymes, and the synthesis of acetate from $\mathrm{CO}_{2}$. J. Bacteriol. 1973, 114, 743-751. [CrossRef]

38. Basra, S.; Anand, B.S. Definition, epidemiology and magnitude of alcoholic hepatitis. World J. Hepatol. 2011, 3, 108-113. [CrossRef]

39. Maddrey, W.C.; Boitnott, J.K.; Bedine, M.S.; Weber, F.L.; Mezey, E.; White, R.I. Corticosteroid therapy of alcoholic hepatitis. Gastroenterology 1978, 75, 193-199. [CrossRef]

(C) 2020 by the authors. Licensee MDPI, Basel, Switzerland. This article is an open access article distributed under the terms and conditions of the Creative Commons Attribution (CC BY) license (http://creativecommons.org/licenses/by/4.0/). 